\title{
Development and validation of a nomogram in survival prediction among advanced breast cancer patients
}

\author{
Jianli Zhao ${ }^{1,2 \#}$, Yaping Yang ${ }^{1,2 \#}$, Danmei Pang ${ }^{3 \#}$, Yunfang Yu ${ }^{1,4}$, Xiao Lin ${ }^{1,2}$, Kai Chen ${ }^{1,2}$, Guolin Ye \\ Jun Tang ${ }^{6}$, Qian $\mathrm{Hu}^{3}$, Jie Chai ${ }^{1,2}$, Zhuofei Bi ${ }^{1,7}$, Linxiaoxiao Ding ${ }^{1,2}$, Wenjing $\mathrm{Wu}^{1,2}$, Yinduo Zeng ${ }^{1,2}$, \\ Xiujuan Guii ${ }^{1,2}$, Donggeng Liu ${ }^{2,8}$, Herui Yao ${ }^{1,2,4}$, Ying Wang ${ }^{1,2}$
}

${ }^{1}$ Guangdong Provincial Key Laboratory of Malignant Tumor Epigenetics and Gene Regulation, Guangzhou Regenerative Medicine and Health Guangdong Laboratory, Sun Yat-sen Memorial Hospital, Sun Yat-sen University, Guangzhou, China; ${ }^{2}$ Breast Tumor Center, Sun Yat-sen Memorial Hospital, Sun Yat-sen University, Guangzhou, China; ${ }^{3}$ Department of Breast Cancer Oncology. Foshan the First Hospital, Sun Yat-sen University, Foshan, China; ${ }^{4}$ Department of Medical Oncology, Sun Yat-sen Memorial Hospital, Sun Yat-sen University, Guangzhou, China; ${ }^{5}$ Department of Breast Surgery. Foshan the First Hospital, Sun Yat-sen University, Foshan, China; ${ }^{6}$ Department of Breast Surgery, Sun Yat-sen University Cancer Center, Guangzhou, China; ${ }^{7}$ Department of Radiation Oncology, Sun Yat-sen Memorial Hospital, Sun Yat-sen University, Guangzhou, China; ${ }^{8}$ Department of Medical Oncology, Sun Yat-sen University Cancer Center, Guangzhou, China

Contributions: (I) Conception and design: Y Wang, J Zhao, D Pang; (II) Administrative support: Y Wang; (III) Provision of study materials or patients: Y Wang, H Yao, D Pang, D Liu, G Ye, J Tang; (IV) Collection and assembly of data: All authors; (V) Data analysis and interpretation: J Zhao, Y Yang, Y Yu; (VI) Manuscript writing: All authors; (VII) Final approval of manuscript: All authors.

"These authors contributed equally and should be considered co-first authors.

Correspondence to: Prof. Dr. Ying Wang. Guangdong Provincial Key Laboratory of Malignant Tumor Epigenetics and Gene Regulation, Breast Tumor Center, Sun Yat-sen Memorial Hospital, Sun Yat-sen University, No. 107 Yanjiang West Road, Guangzhou 510120, China. Email: wangy556@mail.sysu.edu.cn; Prof. Dr. Herui Yao. Guangdong Provincial Key Laboratory of Malignant Tumor Epigenetics and Gene Regulation, Breast Tumor Center, Department of Medical Oncology, Phase I Clinical Trial Centre, Sun Yat-sen Memorial Hospital, Sun Yat-sen University, No. 107 Yanjiang West Road, Guangzhou 510120, China. Email: yaoherui@mail.sysu.edu.cn.

Background: The overall survival (OS) among patients with advanced breast cancer (ABC) varies greatly. Although molecular subtype is known as the most important factor in OS differentiation, significant differences in OS among patients with the same molecular subtype still occur, leading to the need for a more accurate prognostic prediction model. This study aimed to develop a prediction model (nomogram) based on current diagnosis and treatment to predict the OS of newly diagnosed $\mathrm{ABC}$ patients in China.

Methods: From the institution's database, we collected data of 368 ABC patients from Sun Yat-sen Memorial Hospital (national hospital) as a training set to establish a nomogram with prognostic risk factors that calculated the predicted probability of survival. Nomograms were independently validated with 278 patients with $\mathrm{ABC}$ from two other institutions using the concordance index (C-index), calibration plots and risk group stratifications.

Results: The initial primary tumor stage, molecular subtype, disease-free survival (DFS), presence of brain metastasis, and the tumor burden of metastasis disease (local recurrence, oligo-metastatic disease, or multiple-metastatic disease) were included in the prognostic nomogram. The nomogram had a C-index of 0.77 and 0.71 in the training and the validation sets, respectively. The nomogram was able to stratify patients into different risk groups, respectively (HR 6.81, 95\% CI: 4.69 to 9.89, $\mathrm{P}<0.001$ ). In the lower risk score group (risk score $<11$ ), there was no significant difference between the OS with chemotherapy and hormone therapy (HR 0.81, 95\% CI: 0.44 to $1.47, \mathrm{P}=0.48$ ).

Conclusions: We have constructed a novel prediction nomogram that can guide the physicians to select personalized treatment options. Furthermore, our study is the first to add oligo-metastatic disease and primary endocrine/trastuzumab resistance into the prognostic models. 
Keywords: Advanced breast cancer (ABC); prediction model; oligo-metastatic disease; primary endocrine/ trastuzumab resistance

Submitted Apr 23, 2020. Accepted for publication Aug 12, 2020.

doi: $10.21037 /$ atm-20-3473

View this article at: http://dx.doi.org/10.21037/atm-20-3473

\section{Introduction}

About $20-30 \%$ of breast cancer patients ultimately develop metastasis, namely $\mathrm{ABC}$ patients $(1,2)$. The survival time of $A B C$ varies greatly (3), although molecular subtype is known as the most important factor in differentiating the OS, there are still significant differences in survival time among patients with the same molecular subtype. For example, the OS of patients with human epidermal growth factor receptor 2 (HER2) positive $\mathrm{ABC}$ range from less than 10 months to more than 70 months (4). Therefore, a more accurate prognostic prediction model is needed. Accurate predictions of OS are crucial for providing required prognostic information for $\mathrm{ABC}$ patients, guiding oncologists when considering appropriate treatment selection, and stratifying patients for prospective clinical trials.

Previous reports have explored the prediction model of metastasis risk or survival of early breast cancer (EBC) $(5,6)$. The risk factors related to prognosis mainly include, family history of breast cancer, tumor location, number of positive lymph nodes, histological grade, serum CEA, CA125, and CA153, age, tumor size, molecular subtype, and lymphovascular invasion (LVI). However, what is the difference between survival related variables of advanced breast cancer $(\mathrm{ABC})$ and variables of EBC. Moreover, several studies have reported that in patients with $\mathrm{ABC}$, many common parameters such as age, disease-free survival (DFS), hormone receptor status, and burdens of metastasis are significant and independent predictors of OS $(7,8)$.

Additionally, with the development of new drugs, there are more options for the treatment of $\mathrm{ABC}$, such as dual HER2 blockade for HER2 positive ABC (9), PD-1/PD-L1 antibody for advanced triple negative breast cancer (9), and priority endocrine therapy including CDK4/6 inhibitors for hormone receptor positive $\mathrm{ABC}$ (10). However, in clinical practice, there are still $\mathrm{HR}+\mathrm{ABC}$ patients without efficacy under endocrine therapy. Therefore, it is necessary to select suitable $\mathrm{ABC}$ patients with priority endocrine therapy through appropriate prediction models. As such, new chemotherapeutic agents, novel hormonal drugs, and the modern molecular targeted therapies have improved the prognosis to a greater extent but yet to be included as predictors of OS. Besides, some new risk factors, like oligometastatic disease, primary endocrine resistance, primary trastuzumab resistance have never been applies into the prediction model. To date, there is yet to be a generally accepted model based on modern diagnosis and treatment to screen for potential long-term survivors, especially for patients in China.

This study aimed to develop and validate nomogram to predict the OS of newly diagnosed ABC patients in China. Our target was to supply patients and oncologists with precise evaluation of expected survival that would generate superior informed clinical decision-making and would optimize the stratification of patients in future clinical trials. We present the following article in accordance with the STROBE reporting checklist (available at http://dx.doi. org/10.21037/atm-20-3473).

\section{Methods}

\section{Study design and patients}

We conducted this article in accordance with the STROBE guideline checklist. To establish a prognostic model of $\mathrm{ABC}$, we obtained clinical data of 368 breast cancer patients who underwent ABC management in Sun Yatsen Memorial Hospital (national hospital) between January 2006 and December 2016. In this study, inclusion criteria are as follows: (I) female patients aged 18 years or above; (II) patients diagnosed with $\mathrm{ABC}$; (III) patients with available follow-up data; (IV) patients with complete pathological information; and $(\mathrm{V})$ the follow-up time of the patients need to be more than 24 months. The exclusion criteria included the following: (I) prior diagnosis of malignant tumor; (II) male breast cancer patients; (III) follow-up months less than 24 months; (IV) unknown bone, liver, lung or brain metastatic status; (V) unknown estrogen receptor (ER), 
progesterone receptor (PR), HER2 status or tumor stage; (VI) unknown DFS or post-metastasis OS. The study was conducted in accordance with the Declaration of Helsinki (as revised in 2013). The study was approved by the ethics committee of Sun Yat-sen Memorial Hospital, Sun Yatsen University (No. SYSEC-KY-KS-2018-018). Individual informed consent was waived because of the retrospective nature of the study and the anonymity of individual patient data.

The following data were collected for each patient: age of diagnosis, primary tumor site, tumor grade, primary tumor stage, ER status, PR status, HER2 status, the recurrence or metastatic sites (local recurrence/distant lymph nodes/ bone/brain/lung/liver), primary surgery categorization (breast conserving therapy/breast mastectomy), radiation therapy status, chemotherapy, endocrine therapy, survival month, and OS status. DFS was defined as the time from the diagnosis of primary breast cancer to the date of the first local recurrence or distant metastases. We divided DFS into three groups, less than 24 months, 24 to 48 months, and more than 48 months, since 48 -month is the cut-off of primary endocrine/trastuzumab resistance and secondary endocrine/trastuzumab resistance. According to previous studies, primary endocrine or trastuzumab resistance $\mathrm{ABC}$ patients showed worse benefit from treatment and were equal to first-line-treatment-failure patients $(11,12)$. Postmetastasis OS was measured from first recurrence or metastasis to the date of death from any cause or to most recent follow-up date.

There were 732 ABC patients diagnosed in Sun Yatsen Memorial Hospital, Foshan the First Hospital, and Sun Yat-sen University Cancer Center. Among them, 51 patients were excluded because they did not meet the inclusion criteria, and another 35 patients were excluded because of the lack of survival data. A total of 646 patients were included in the final analysis, 368 of them were diagnosed in Sun Yat-sen Memorial Hospital, set as training data, 278 patients were set as validation data in Foshan the First Hospital, and Sun Yat-sen University Cancer Center. Among the validation cohort, 180 patients were from Foshan the First Hospital, while the other 98 patients were from Sun Yat-sen University Cancer Center, and they were all diagnosed ABC between Jan 2007 and Dec 2017. Same inclusion and exclusion criteria were used for patient selection in the validation set. The research project had been approved by the ethics committee of Sun Yatsen Memorial Hospital, and all procedures were done in accordance with the Declaration of Helsinki.

\section{Establishment of the Nomogram}

We conducted a descriptive analysis of the baseline clinicopathological features of the included patients and used the Chi-square test to compare the characteristics of patients between the training and validation cohort. In the training set, survival outcome was estimated by the KaplanMeier method, and the univariate and multivariate analyses were generated with a Cox proportional hazard regression. Variables that achieved significance at $\mathrm{P}<0.05$ in univariate analyses were entered into the multivariable analyses. We used the Cox regression model and the "rms" package in $\mathrm{R}$ to develop an OS prediction nomogram with 1-, 2and 3-year OS as the endpoints. We used the C-index to evaluate the discriminative ability of the nomogram, and calibration plots to visualize the agreement between the predicted and actual 1-, 2- and 3-year OS.

\section{Validation of the Nomogram}

The nomogram was validated among 278 breast cancer patients from two other hospitals in China. The prognostic model performance for predicting outcome was generated by $\mathrm{C}$-index and the accuracy of the nomogram was evaluated using calibration plots of 1-, 2- and 3-year OS. The value of the $\mathrm{C}$-index ranges from 0.5 to 1.0 , with 0.5 indicated that the model did not predict risk better than randomized guess, and a score of 1 indicated perfect prediction of risk.

\section{Risk stratification and treatment recommendation based on the Nomogram}

In addition to assessing the accuracy of the nomogram with C-index, we sought to illustrate the discrimination ability of the nomogram by stratifying prognostic risk and treatment recommendation between chemotherapy and hormone therapy. We then proceed to determine the cutoff value through calculation of risk score and distribution of predicted OS.

\section{Statistical analysis}

Univariate and multivariate Cox regression analysis were used to test the independent significance of different factors. The OS was calculated using the Kaplan-Meier method and the log-rank test, and the hazard ratios (HRs) and $95 \%$ confidence intervals $(\mathrm{Cls})$ were calculated using 


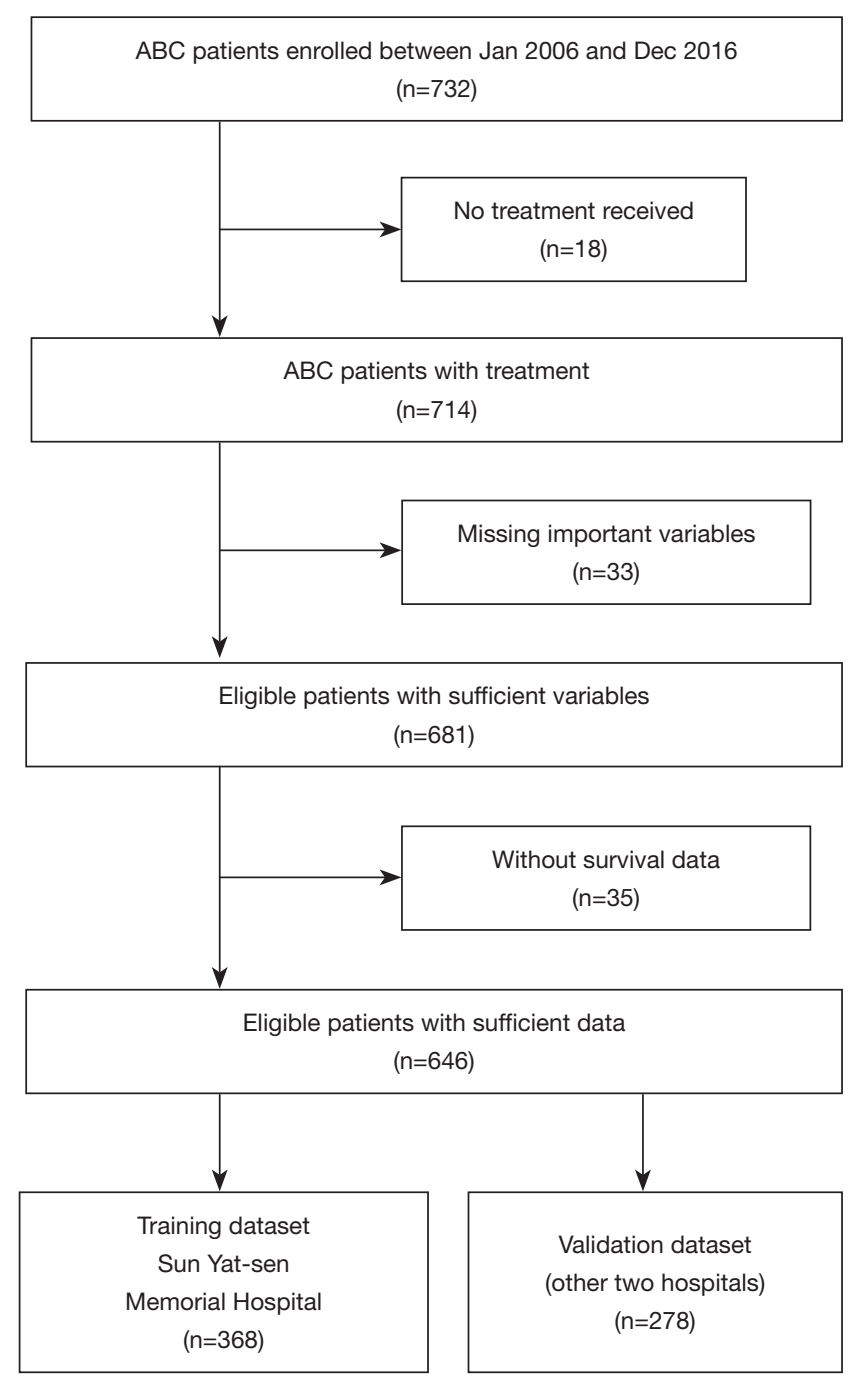

Figure 1 Flow diagram for the eligible patients.

the Cox regression model. The optimal cutoff values for the predictive models that were used to separate patients into low-risk and high-risk groups were generated with the survminer package. For all the analyses, $\mathrm{P}$ values less than 0.05 were considered statistically significant. The statistical analyses were conducted in SPSS 23.0 (SPSS, Chicago, IL) and R (version 3.3.2, http://www.r-project.org).

\section{Results}

\section{Clinicopatbologic characteristics of patients}

There were 732 ABC patients diagnosed in Sun Yat-sen Memorial Hospital, Foshan the First Hospital, and Sun Yatsen University Cancer Center. Among them, 51 patients were excluded because they did not meet the inclusion criteria, and another 35 patients were excluded because of the lack of survival data. A total of 646 patients were included in the final analysis, 368 of them were diagnosed in Sun Yat-sen Memorial Hospital, set as training data, 278 patients were set as validation data in Foshan the First Hospital, and Sun Yat-sen University Cancer Center (Figure 1).

All patients in the training and validation cohort were $\mathrm{ABC}$ patients. In the training cohort patients who had missing values on any of the examined variables, including the initial primary tumor stage, the molecular subtype of primary tumor, DFS, presence of brain metastasis, and the tumor burden of metastasis disease were excluded. Thus, a total of 368 patients were included according to the inclusion criteria and excluding criteria, and 180 patients died in the training dataset. The validation dataset consisted of 278 patients, among which, 67 patients died. The clinicopathological characteristics of patients in the training and validation cohorts are summarized in Table 1 .

\section{Prognostic factors of OS in training cohort}

The characters of primary tumor, like the tumor stage and molecular subtype which was defined by ER status, PR status and HER2 status, were significantly associated with OS. In addition, the clinical features of first recurrence or metastasis were also crucial in predicting OS. The DFS was defined as the time from the diagnosis of primary breast cancer to the date of the first local recurrence or distant metastases. We divided DFS into three groups, less than 24 months, 24 to 48 months, and more than 48 months. Since 48-month is the cut-off of primary endocrine/ trastuzumab resistance and secondary endocrine/ trastuzumab resistance, therefore, the DFS, the primary endocrine or trastuzumab resistance and presence of brain metastasis were also factors that had crucial impact on OS. Interestingly, the age of patient first diagnosed with breast cancer, BMI, menstruation status, and ECOG (Eastern Cooperative Oncology Group performance) status was not significantly associated with OS (Table 2).

\section{Multivariable analysis and prognostic nomogram for OS}

Cox proportional regression model showed primary tumor characteristics as tumor stage (0.045), molecular subtype $(<0.001)$, and clinical features of recurrence or metastasis including DFS (0.043), tumor burden of metastasis $(<0.001)$ and presence of brain metastasis $(0.042)$ were independent 
Table 1 Demographics and clinicopathologic characteristics of the training cohort and the validation cohort

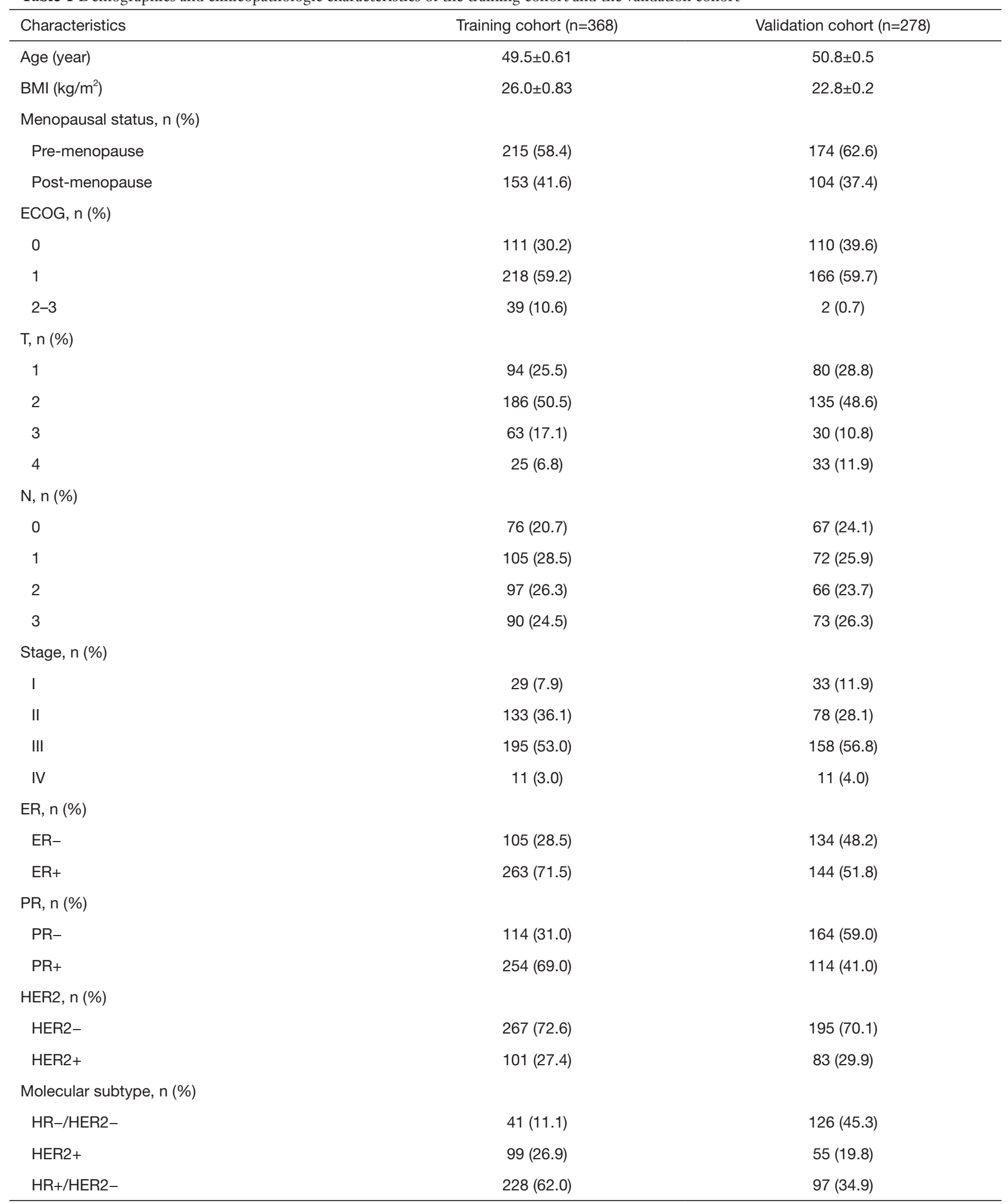

Table 1 (continued) 
Table 1 (continued)

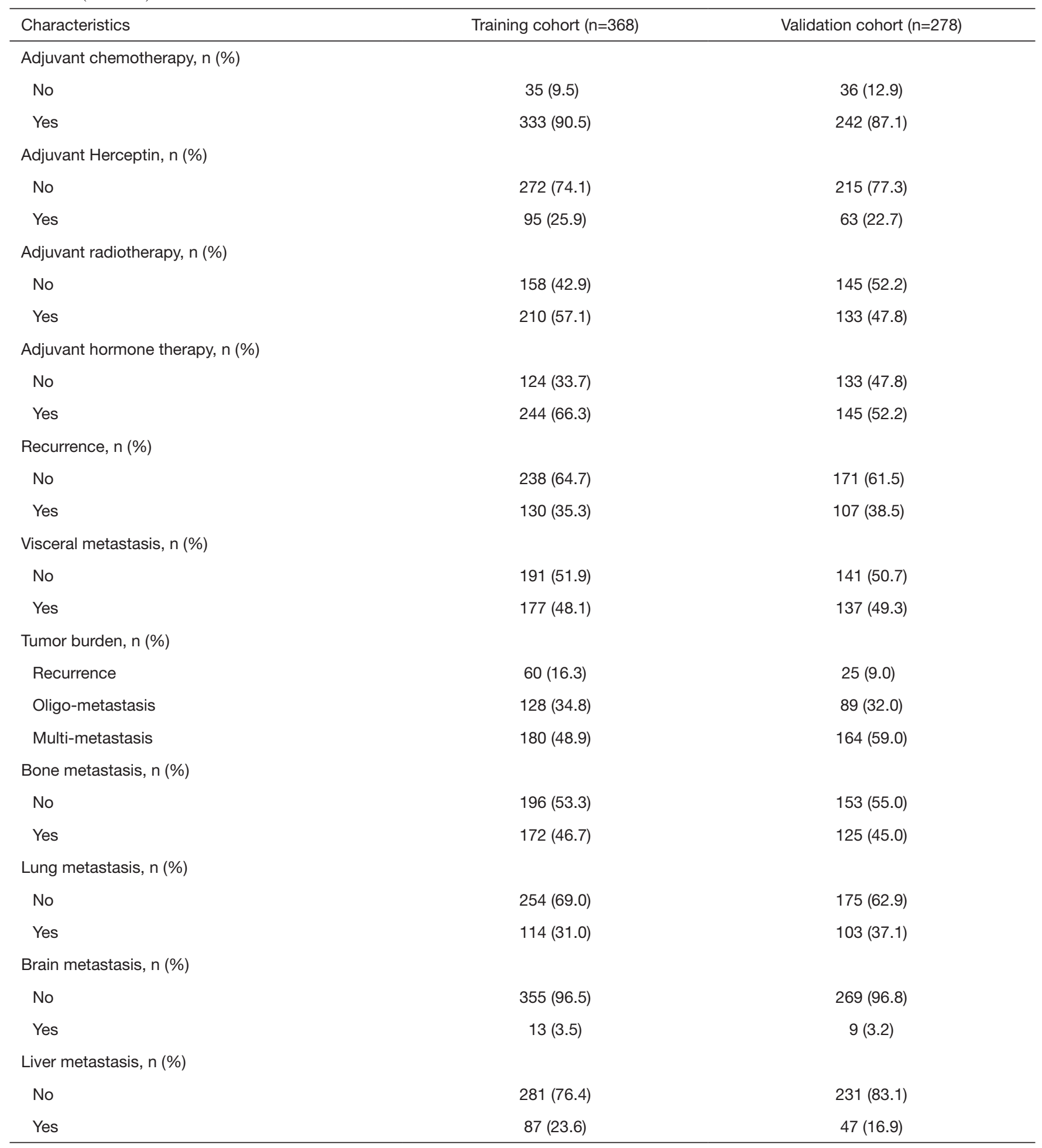

DFS, disease free survival; tumor burden, the tumor burden of metastatic disease, as recurrence, oligo-metastasis, and multi-metastasis; $\mathrm{T}$, the size of primary tumor; N, the number of involved lymph nodes; ECOG status, Eastern Cooperative Oncology Group performance status; ER, estrogen receptor; PR, progesterone receptor; HER2, human epidermal growth factor receptor 2; HR, hormone receptor. 
Table 2 The univariable analysis of prognostic risk factors

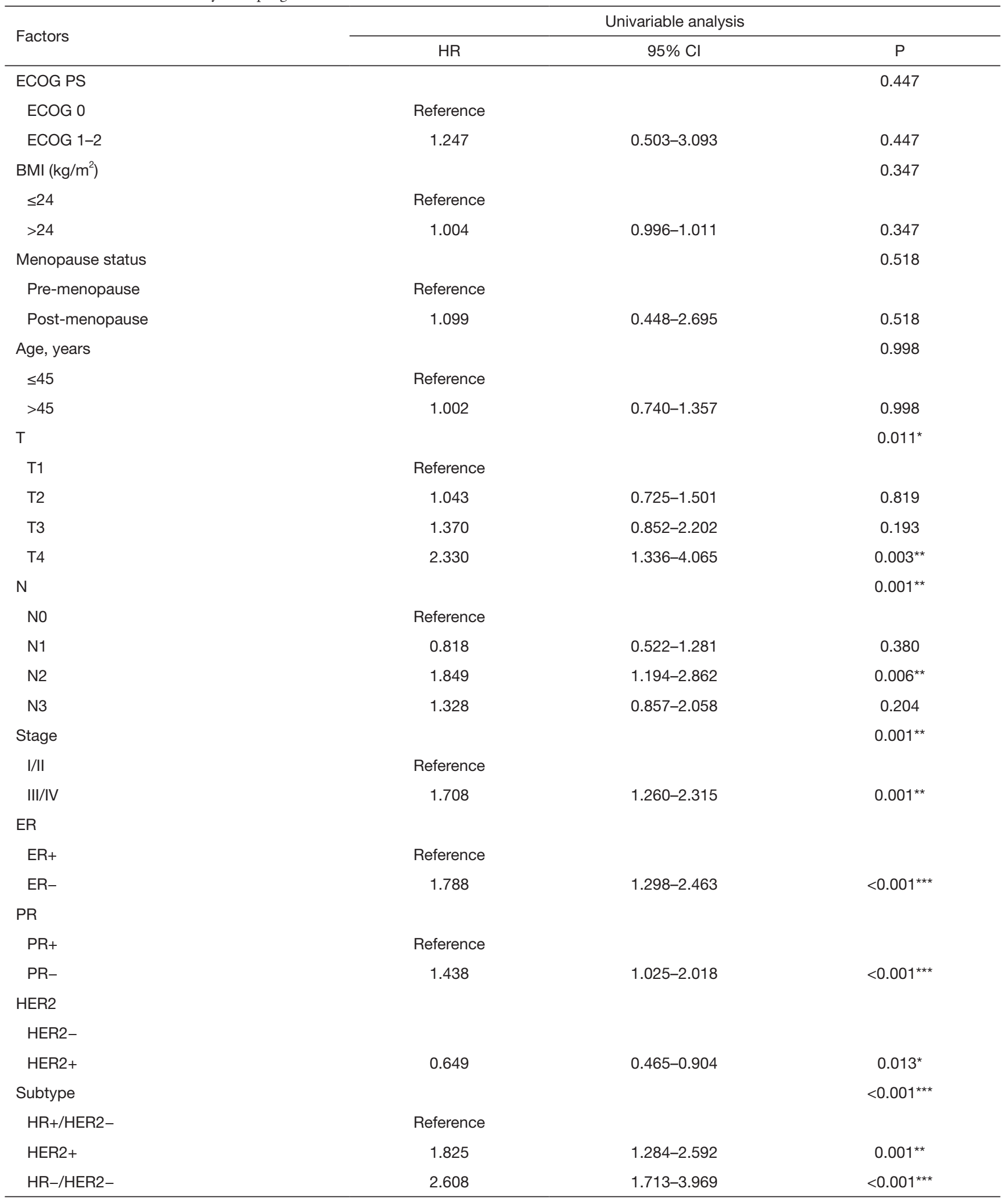

Table 2 (continued) 
Table 2 (continued)

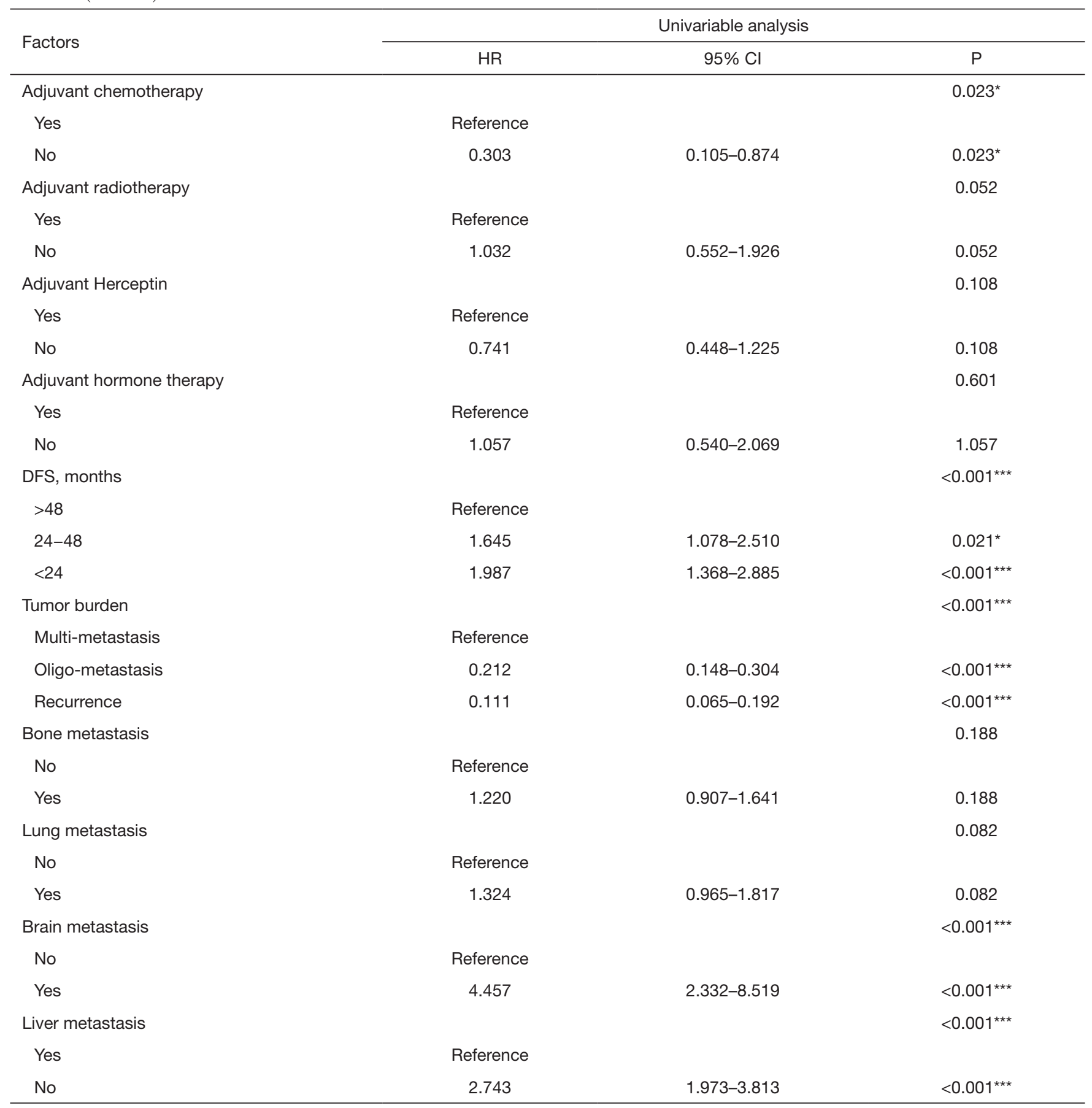

${ }^{*}, \mathrm{P}<0.05 ;{ }^{* *}, \mathrm{P}<0.01 ;{ }^{* *}, \mathrm{P}<0.001$. DFS, disease free survival; tumor burden, the tumor burden of metastatic disease, as recurrence, oligometastasis, and multi-metastasis; T, the size of primary tumor; N, the number of involved lymph nodes; ECOG status, Eastern Cooperative Oncology Group performance status; ER, estrogen receptor; PR, progesterone receptor; HER2, human epidermal growth factor receptor 2; HR, hormone receptor. 
Table 3 The Cox proportional hazards regression analysis

\begin{tabular}{|c|c|c|c|}
\hline Factors & \multicolumn{3}{|c|}{ Selected factors for building the Nomogram } \\
\hline Stage & & & $0.045^{\star}$ \\
\hline $1 / I 1$ & Reference & & \\
\hline III/IV & 1.266 & $1.061-1.547$ & $0.045^{\star}$ \\
\hline HR+/HER2- & Reference & & \\
\hline HER2+ & 1.503 & $1.037-2.178$ & $0.031^{*}$ \\
\hline HR-/HER2- & 3.079 & $1.993-4.757$ & $<0.001^{\star \star \star}$ \\
\hline DFS, months & & & $0.043^{*}$ \\
\hline$<24$ & 1.634 & $1.110-2.406$ & $0.013^{*}$ \\
\hline Tumor burden & & & $<0.001^{\star * \star}$ \\
\hline Multi-metastasis & Reference & & \\
\hline Oligo-metastasis & 0.227 & $0.061-0.184$ & $<0.001^{\star \star \star}$ \\
\hline Recurrence & 0.106 & $0.061-0.184$ & $<0.001^{\star \star \star}$ \\
\hline Brain metastasis & & & $0.042^{*}$ \\
\hline No & Reference & & \\
\hline Yes & 2.006 & $1.027-3.917$ & $0.042^{*}$ \\
\hline
\end{tabular}

${ }^{*}, \mathrm{P}<0.05 ;{ }^{* \star}, \mathrm{P}<0.01 ;{ }^{* \star}, \mathrm{P}<0.001$. DFS, disease free survival; tumor burden, the tumor burden of metastatic disease, as recurrence, oligometastasis, and multi-metastasis; T, the size of primary tumor; $\mathrm{N}$, the number of involved lymph nodes; ECOG status, Eastern Cooperative Oncology Group performance status; ER, estrogen receptor; PR, progesterone receptor; HER2, human epidermal growth factor receptor 2; HR, hormone receptor.

predictors of OS (Table 3). A nomogram that incorporated these significant prognostic factors was established (Figure 2, Table 4). The nomogram illustrated the tumor burden of metastasis had the largest contribution to prognosis, followed by the presence of brain metastasis and molecular subtype of primary tumor. The initial tumor stage and DFS showed a moderate impact on the OS. Each subtype with these variables was assigned a score on the point scale, as calculated by the rms software. By adding up these scores and locating it on the total point scale, we were able to estimate the probability of survival at each time point (Figure 2).

\section{Calibration and validation of the Nomogram}

The C-index of the prognostic nomogram were 0.77 (95\% CI: $0.71-0.84)$ and 0.71 (95\% CI: 0.60-0.82) in the training and the validation cohort, respectively. The calibration plots suggested that the accuracy of the predicted 1-, 2and 3-year OS was excellent in both the training cohort (Figure 3) and validation cohort (Figure 4).

\section{Performance of the Nomogram in risk stratification and recommendation on management choice of chemotherapy and bormone therapy}

The distribution of the predicted OS by the nomogram was shown in Figure 5, and we assigned the patients into different risk sub-group by the cut-off value of 11 , respectively (HR 6.81, 95\% CI: 4.69 to $9.89, \mathrm{P}<0.001$ ). The validation dataset and the combination cohort of training and validation dataset could also be divided into different risk subgroup by the cut-off value (Figures S1,S2). Moreover, in the lower risk score group (risk score $<11$ ), there was no significant difference between the OS with 
Table 4 Point assignment and prognostic score

\begin{tabular}{ll}
\hline Variables & Score \\
\hline Tumor stage & 0 \\
Stage I/II & 1 \\
Stage III/IV & \\
Subtype & 0 \\
HR+/HER2- & 2 \\
HER2+ & 4 \\
HR-/HER2- & \\
DFS, months & \\
$>48$ & 1 \\
$24-48$ & 2 \\
$<24$ & 10 \\
Tumor burden & 5 \\
Multi-metastasis & 0 \\
Oligo-metastasis & \\
Recurrence & \\
Brain metastasis & \\
No & \\
Yes & \\
\hline
\end{tabular}

DFS, disease free survival; tumor burden, the tumor burden of metastatic disease, as recurrence, oligo-metastasis, and multi-metastasis; $\mathrm{T}$, the size of primary tumor; $\mathrm{N}$, the number of involved lymph nodes; ECOG status, Eastern Cooperative Oncology Group performance status; ER, estrogen receptor; $\mathrm{PR}$, progesterone receptor; HER2, human epidermal growth factor receptor 2; HR, hormone receptor.

chemotherapy and hormone therapy (HR 0.81, 95\% CI: 0.44 to $1.47, \mathrm{P}=0.48$ ) (Figure 6 ). In view of the larger adverse effects of chemotherapy, hormone therapy should be recommended as the first line therapy in the lower risk group.

\section{Discussion}

We have constructed a novel prediction nomogram that can guide the physicians to select personalized treatment options and also help patients to obtain more clear insights into their future outcomes. For patients with $\mathrm{ABC}$, more than $90 \%$ of patient regarded the expected survival as the most valuable information for informed decision-making. Therefore $(13,14)$, accurate and personalized details of OS can help patients to be mentally prepared (15). Moreover, a reliable prediction of the $\mathrm{OS}$ in $\mathrm{ABC}$ patients is a vital basis for optimal therapy selection.

Although a few previous models have been developed to predict the prognosis of $\mathrm{ABC}$ patients, current guidelines lack clear recommendations for uniform or universally accepted prediction model. Llombart-Cussac et al. developed a prognostic model using data from $\mathrm{ABC}$ patients treated with first-line chemotherapy plus bevacizumab for HER2negative $A B C$ (16). However, important information on the duration and efficacy of adjuvant hormonal therapy was not available and the influence of previous endocrine therapy on the outcome cannot be evaluated. Similar weaknesses were also found in a cohort analysis in Germany, in which accepted prognostic factors such as HER2/neu (an accepted prognostic factor) was not considered in the model (17). Lee et al. developed a model that predicts the postmetastasis overall survival (PMOS) based on patients who developed distant metastasis during their follow-up after the initial treatment from the Seoul National University Hospital. However, the classification of the Ki-67 staining for $\mathrm{ABC}$ patients was not determined by the absolute value, which resulted in inaccessibility of the impact of molecular subtype on prognosis (18). Another nomogram was constructed from patients treated only with anthracyclines, CMF, or capecitabine as first-line chemotherapy (19).

Today, patients with $\mathrm{ABC}$ are given a larger number of choices in treatment options, including new drugs (Eribulin), endocrine treatment (Fulvestrant), molecular targeted therapies (Palbociclib, Pertuzumab) and small tyrosine-kinase inhibitors (Neratinib). As a result, the past nomogram may have underestimated the expected OS of patients treated with current chemotherapeutic and biological agents (20-23). Therefore, the validations of the existing prediction models for $\mathrm{ABC}$ showed a modest result with poor c-statistics ranging from 0.50 to 0.63 (24). These inadequate prediction models indicate that the variables in the former prognostic models were insufficient, and better risk factors were needed to increase the robustness and discriminatory abilities of the model.

Recent studies found that a distinctive subset of $\mathrm{ABC}$ such as oligometastatic disease, which is defined as low volume metastatic disease with limited number and size of metastatic lesions (up to five and not necessarily in the same organ), can achieve rapid disease control and long-term survival benefit $(25,26)$. Besides, the advent of new drugs has also greatly improved the OS of ABC patients $(11,27-29)$. In patients with HR+/HER2- ABC subtype, Fulvestrant 


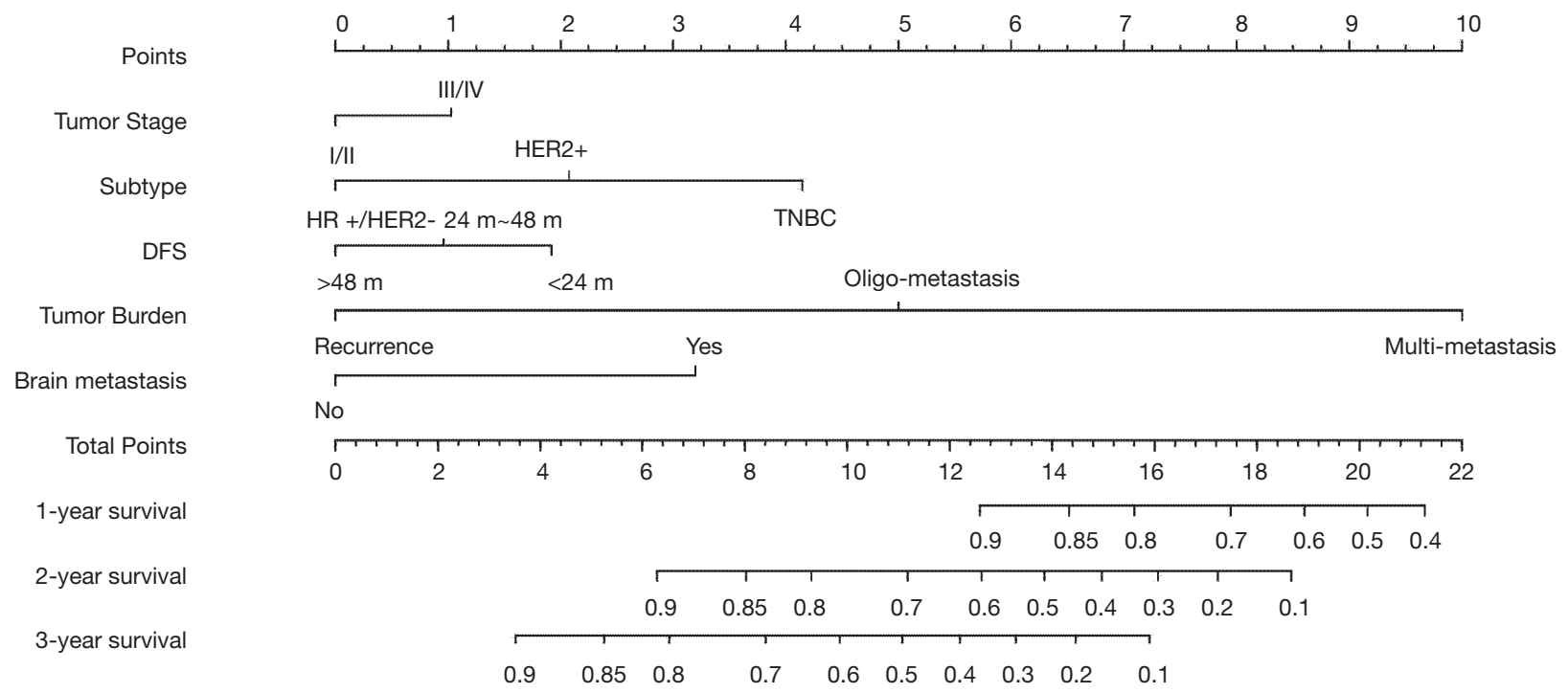

Figure 2 The prognostic nomogram to predict the 1-year, 2-year and 3-year OS of ABC patients. For each patient, we calculated the points of the clinicopathological features, and summed up the points to obtain the total points. The predicted 1-, 2- and 3-year OS can be estimated based on the total points of each patient. Tumor stage, the stage of primary tumor; Subtype, the molecular subtype of primary tumor; DFS, disease free survival; tumor burden, the tumor burden of metastatic disease, as recurrence, oligo-metastasis and multi-metastasis; brain metastasis, the presence of brain metastasis; T, the size of primary tumor; N, the number of involved lymph nodes; ER, estrogen receptor; PR, progesterone receptor; HER2, human epidermal growth factor receptor 2; HR, hormone receptor.

yielded significantly longer OS as the first-line therapy (54.1 months) compared to anastrozole (48.4 months) in the Phase II FIRST study (30). Phase III CLEOPATRA study also reported a significant improvement in OS (HR 0.66; 95\% CI: 0.52-0.84; $\mathrm{P}=0.0008$ ) with Pertuzumab, Trastuzumab, and Docetaxel as first-line treatment in patients with HER2 + subtype ABC, compared with the use of a single HER2-targeted agent (4).

Besides, for patients with triple negative breast cancer, the inhibitor of immune checkpoint PD-L1, Atezolizumb combined with Nano-Paclitaxel, showed a significantly increased PFS and a trend of longer OS (25.0 vs. 15.5 months) in PD-L1 positive patients in the Impassion 130 study (31). Hence, a more efficient prognostic model composed of new risk factors and new cohort of patients with current breast cancer management is needed for choosing patients for optimal therapeutic schedule without imposing unnecessary side effects.

In order to improve the performance of the model, we optimized the design and constructed a novel prognostic nomogram based on multi-center real-world data to predict the OS of newly diagnosed $\mathrm{ABC}$ patients, and validated the clinical value of our prognostic model in an independent validation dataset. We included crucial prognostic parameters such as initial primary tumor stage, the precise molecular subtype (ER, PR, HER2/neu, and Ki-67 staining determined by absolute value), the details and effect of adjuvant therapy, the tumor burden of metastasis disease (local recurrence, oligo-metastatic disease, or multiplemetastatic disease), modern therapeutic regimen (cytotoxic, endocrine, and molecular targeted therapies), and others as indicated in Tables 1,2. Our data indicated that the initial primary tumor stage $(\mathrm{P}=0.045)$, molecular subtype $(\mathrm{P}<0.001)$, DFS $(\mathrm{P}=0.043)$, presence of brain metastasis $(\mathrm{P}=0.042)$ showed significant association with OS. Interestingly, in this study, we found that the tumor burden of metastasis disease (local recurrence, oligo-metastatic disease, or multiple-metastatic disease, $\mathrm{P}<0.001$ ), were significantly associated with OS but was not considered as risk factors in previous nomograms, and are firstly introduced as risk factors in our prognostic nomogram.

Previous survival prediction models often used liver metastasis, lung metastasis, or bone metastasis as one of the variables, however, our study found that the number of metastases is a more important prognostic factor than the location of metastasis. Oligo-metastatic disease is often 


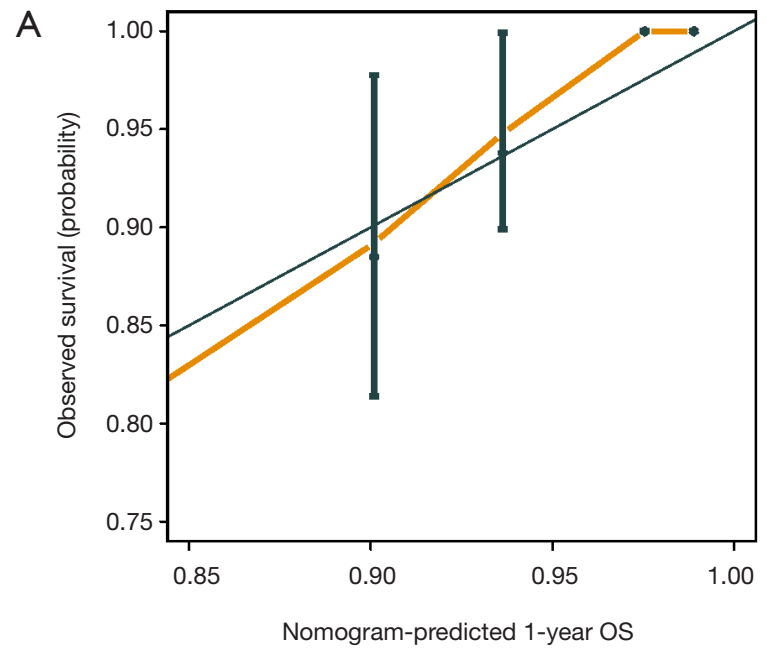

B

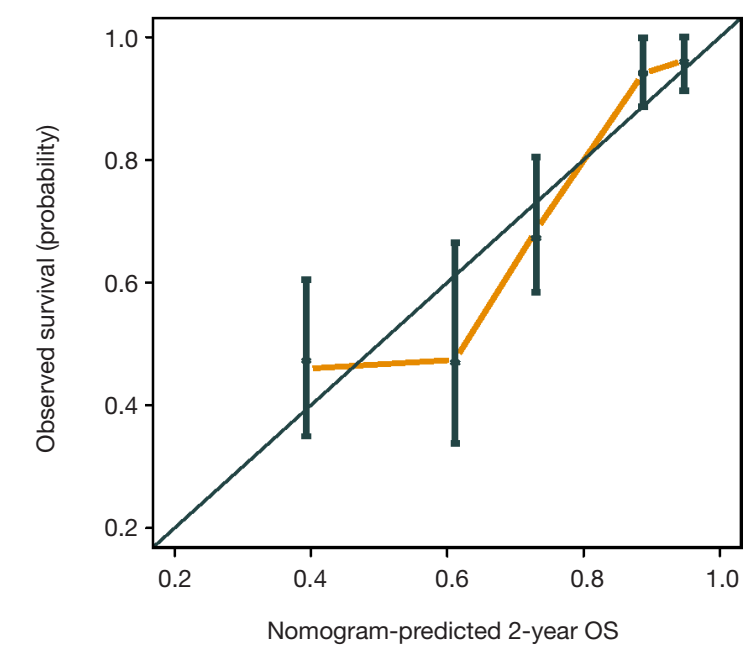

C

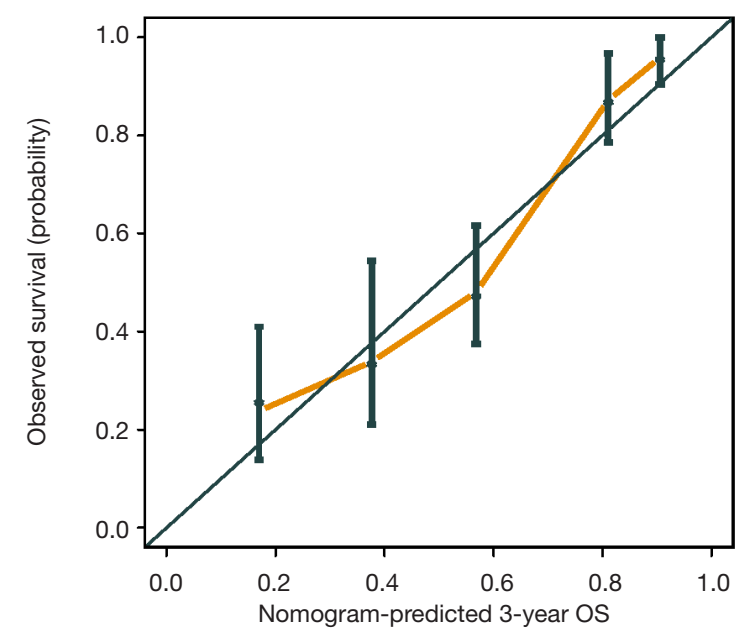

Figure 3 The calibration curves for predicting patient survival at 1-year OS (A), 2-year (B), 3-year (C) in the training cohort.
A

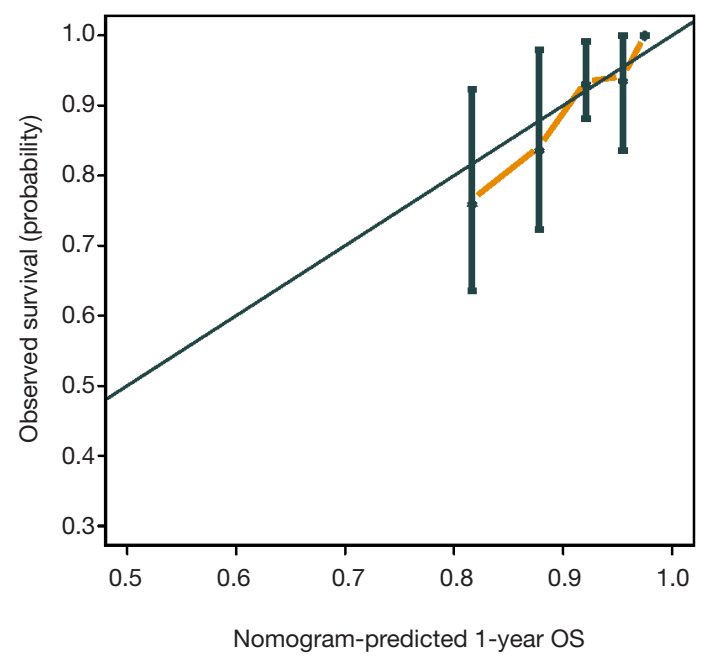

B

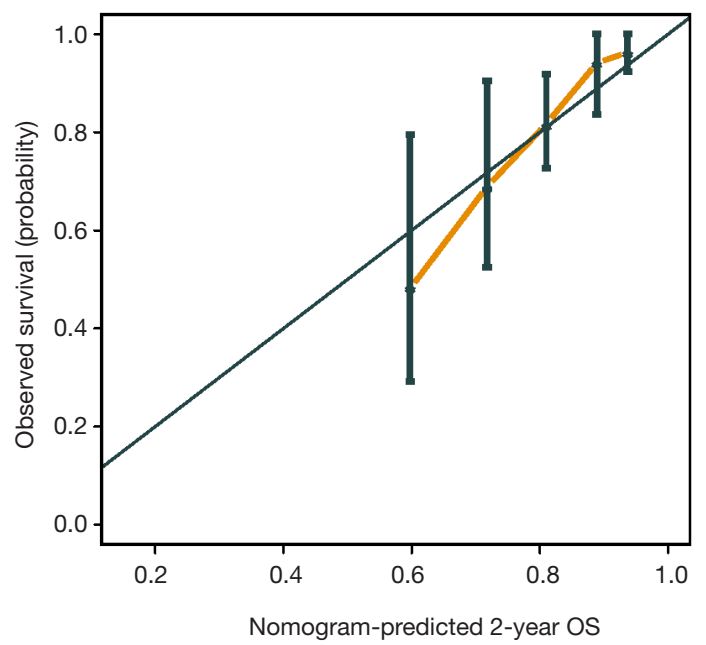

C

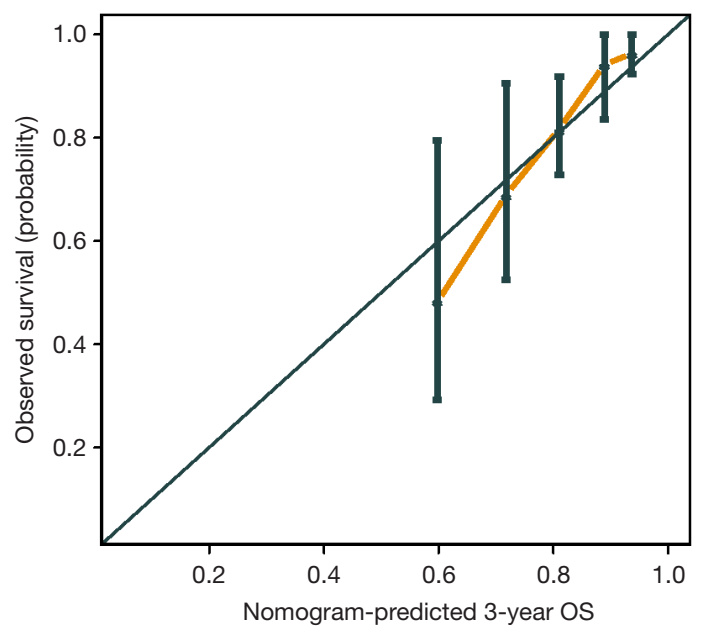

Figure 4 The calibration curves for predicting patient survival at 1-year OS (A), 2-year (B), 3-year (C) in the validation cohort. 


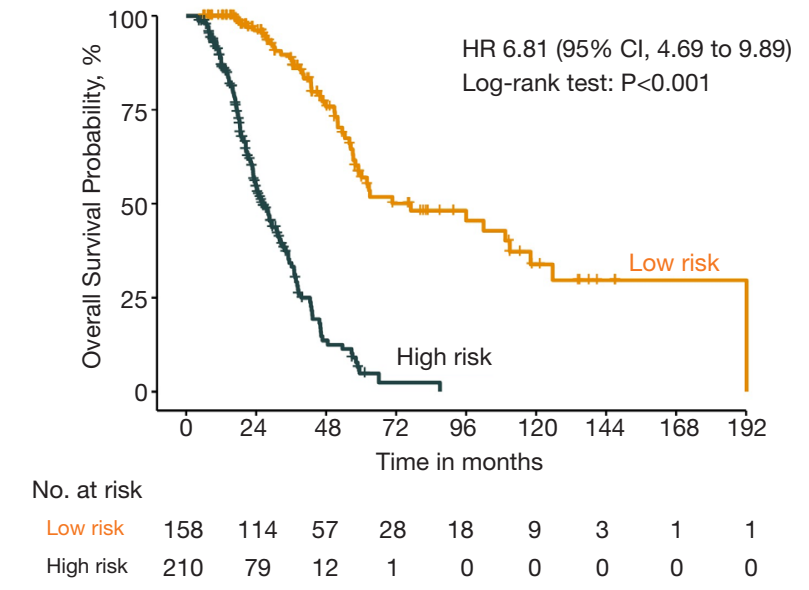

Figure 5 The OS of the training cohort was divided into two significant different groups by the cutoff. The cutoff of risk score is determined to be 11 . Patient's risk score above 11 indicated worse prognosis.

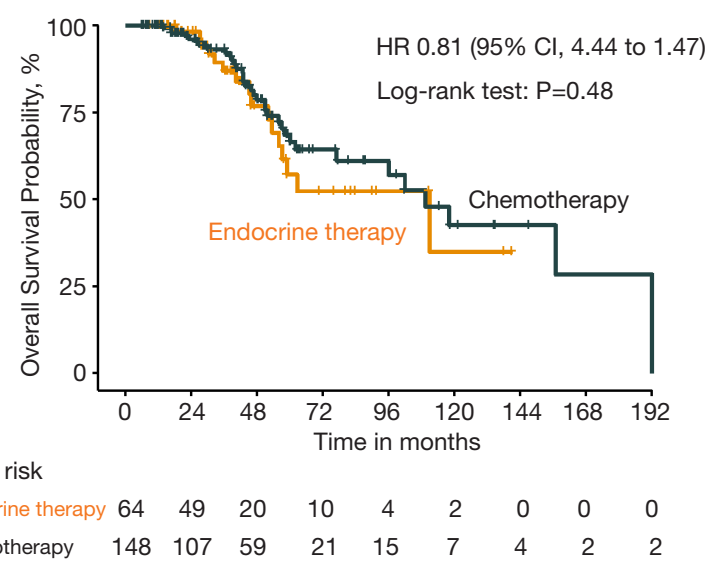

Figure 6 The OS between chemotherapy and hormone therapy in the lower risk group of HR+/HER2- patients. In the lower risk group of $\mathrm{HR}+/ \mathrm{HER} 2-\mathrm{ABC}$ patients, the OS between chemotherapy and hormone therapy showed no significant difference $(\mathrm{P}=0.48)$.

overlooked in the inclusion of risk factors in previous nomogram development. However, the factor of oligometastatic disease had both number and size information of metastatic lesions, and was growing into a vital risk factor of ABC. Therefore, in this study, visceral metastasis was replaced with oligo-metastatic disease, which resulted in a better predictive effect. Our finding was consistent with several previous retrospective studies, which reported that oligometastatic disease of $\mathrm{ABC}$ patients might benefit from locoregional treatment of the primary tumor and metastatic sites strategies to achieve long-lasting remission and achieve long-term survival $(29,32)$.

Meanwhile, prospective Phase III MF07-01 trial also revealed statistically significant improvement in OS with locoregional treatment (46 vs. 37 months) at median 40 months follow up, especially for patients with a more indolent form of $\mathrm{ABC}$ such as solitary bone metastasis (33). To the best of our knowledge, this is the first time that the oligo-metastatic disease is considered as a variable in prognostic models.

In this prognostic model, we divided DFS with a 2-year endpoint, which is the cut-off of primary endocrine/ trastuzumab resistance and secondary endocrine/ trastuzumab resistance. According to previous studies, primary endocrine or trastuzumab resistance $\mathrm{ABC}$ patients showed worse benefit from treatment and were equal to first-line-treatment-failure patients $(11,12)$. In our current study, we also achieved similar conclusion as we found that primary endocrine or trastuzumab resistance showed significant value in prediction of OS. Therefore, our nomogram is the first to introduce the addition of "primary endocrine/trastuzumab resistance" risk factor.

Besides, the development of brain metastasis results in a poor prognosis and a heavily impaired quality of life (34). Similarly, we also found that presence of brain metastasis was significantly associated with worse OS. For early diagnosis of brain metastasis on high risk patients, Genre et al. have validated the Graesslin's nomogram which is able to predict subsequent brain metastasis in patients with proven extra-cerebral metastatic breast cancer (MBC) (21).

In our study, the developed nomogram had a C-index of 0.77 (95\% CI: $0.71-0.84$ ) and 0.71 (95\% CI: 0.60-0.82) in the training and the validation sets, respectively. The predicted survival using the nomogram is well correlated with actual OS, which was indicated by the ideal calibration plots. The above results suggested this nomogram is accurate, reliable and robust, providing good discrimination in both training and validation cohorts. Therefore, the model was able to stratify patients into different risk group. More importantly, in the HR+/HER2- lower risk score group (risk score $<11$ ), there was no significant difference between OS with chemotherapy and hormone therapy (HR 0.81, 95\% CI: 0.44 to $1.47, \mathrm{P}=0.48$ ). Based on our findings, we could strongly suggest hormone therapy in the lower risk group, in view of the increasing unnecessary compromise in quality of life after chemotherapy.

Nevertheless, we are aware of the limitations of this 
study. First of all, patients in both training and validation cohorts were retrospectively selected from independent multi-center databases between 2006 and 2016, due to a retrospective nature, limited available data of patient characteristics were somewhat scattered, and the diagnosis time span of patients was above 10 years, new drugs like Fulvestrant, Pertuzumab, Trastuzumab, Palbociclib, Laptinib, and Nano-Paclitaxel were available for clinical management, but are not included in nomogram prediction, therefore, selection bias was inevitable. The retrospective essence of our study calls for the prospective verification and validation of the prognostic model. Secondly, With the development of precise treatment of $\mathrm{ABC}$, more biomarkers have become important prognostic factors, such as ctDNA, PD-L1 expression, tumor mutation burden (TMB), tumor infiltrating lymphocytes and other biomarkers related to tumor microenvironment, tumor proliferation or tumor metabolism. But the information of these biomarkers was unavailable in this retrospective study. Therefore, further prospective study with molecular profile and targeted therapy related to these biomarkers is required.

\section{Conclusions}

In conclusion, we have constructed a novel prediction nomogram that can guide the physicians to select personalized treatment options and also help patients to obtain a clearer insight into their future outcomes. Furthermore, this nomogram can be a valuable tool to screen suitable patients who might be potential candidates for avoiding chemotherapy in first-line treatment to increase quality of life.

\section{Acknowledgments}

Funding: This study was supported by grants from the National Science and Technology Major Project (2020ZX09201021), the National Natural Science Foundation of China (81802656, 81572596, 81972471, U1601223), the Natural Science Foundation of Guangdong Province (2017A030313828, 2016A030313312), the Guangzhou Science and Technology Major Program (201704020131), the Sun Yat-Sen University Clinical Research 5010 Program (2018007), the Sun Yat-Sen Scientific Research Program (YXQH201909), the Sun YatSen Clinical Research Cultivating Program (SYS-C-201801, SYS-C-202003), the Guangdong Science and Technology Department (2017B030314026).

\section{Footnote}

Reporting Checklist: The authors have completed the STROBE reporting checklist. Available at http://dx.doi. org/10.21037/atm-20-3473

Data Sharing Statement: Available at http://dx.doi. org/10.21037/atm-20-3473

Conflicts of Interest: All authors have completed the ICMJE uniform disclosure form (available at http://dx.doi. org/10.21037/atm-20-3473). The authors have no conflicts of interest to declare.

Ethical Statement: The authors are accountable for all aspects of the work in ensuring that questions related to the accuracy or integrity of any part of the work are appropriately investigated and resolved. The study was conducted in accordance with the Declaration of Helsinki (as revised in 2013). The study was approved by the ethics committee of Sun Yat-sen Memorial Hospital, Sun Yatsen University (No. SYSEC-KY-KS-2018-018). This study is retrospective analysis, and all the individual patient data is anonymous. No patients were involved in setting the research question or the outcome measures, nor were they involved in developing plans for design or implementation of the study. No patients were asked to advise on interpretation or writing up of results. There are no plans to disseminate the results of the research to study participants or the relevant patient community. Therefore, a statement of informed consent of study participants was institutionally waived because of the retrospective nature of the study and the anonymity of individual patient data.

Open Access Statement: This is an Open Access article distributed in accordance with the Creative Commons Attribution-NonCommercial-NoDerivs 4.0 International License (CC BY-NC-ND 4.0), which permits the noncommercial replication and distribution of the article with the strict proviso that no changes or edits are made and the original work is properly cited (including links to both the formal publication through the relevant DOI and the license). See: https://creativecommons.org/licenses/by-nc-nd/4.0/.

\section{References}

1. Bartmann C, Diessner J, Blettner M, et al. Factors influencing the development of visceral metastasis of 
breast cancer: A retrospective multi-center study. Breast 2017;31:66-75.

2. Diessner J, Wischnewsky M, Stüber T, et al. Evaluation of clinical parameters influencing the development of bone metastasis in breast cancer. BMC Cancer 2016;16:307.

3. Pe M, Dorme L, Coens C, et al. Statistical analysis of patient-reported outcome data in randomised controlled trials of locally advanced and metastatic breast cancer: a systematic review. Lancet Oncol 2018;19:e459-69.

4. Swain SM, Baselga J, Kim SB, et al. Pertuzumab, trastuzumab, and docetaxel in HER2-positive metastatic breast cancer. N Engl J Med 2015;372:724-34.

5. Lin Y, Fu F, Lin S, et al. A nomogram prediction for the survival of patients with triple negative breast cancer. Oncotarget 2018;9:32108-18.

6. Wang Y, Yang Y, Chen Z, et al. Development and validation of a novel nomogram for predicting distant metastasis-free survival among breast cancer patients. Ann Transl Med 2019;7:537.

7. Marshall EM, Bertaut A, Desmoulins I, et al. Prognostic Factors of Survival among Women with Metastatic Breast Cancer and Impact of Primary or Secondary Nature of Disease on Survival: A French Population-Based Study. Breast J 2017;23:138-45.

8. Chang E, Mougalian SS, Adelson KB, et al. Association between prolonged metastatic free interval and recurrent metastatic breast cancer survival: findings from the SEER database. Breast Cancer Res Treat 2019;173:209-16.

9. O'Sullivan H, Collins D, O'Reilly S. Atezolizumab and Nab-Paclitaxel in Advanced Triple-Negative Breast Cancer. N Engl J Med 2019;380:986.

10. Turner NC, Slamon DJ, Ro J, et al. Overall Survival with Palbociclib and Fulvestrant in Advanced Breast Cancer. N Engl J Med 2018;379:1926-36.

11. Cardoso F, Senkus E, Costa A, et al. 4th ESO-ESMO International Consensus Guidelines for Advanced Breast Cancer (ABC 4). Ann Oncol 2018;29:1634-57.

12. Wong H, Leung R, Kwong A, et al. Integrating molecular mechanisms and clinical evidence in the management of trastuzumab resistant or refractory HER-2 metastatic breast cancer. Oncologist 2011;16:1535-46.

13. Libert Y, Peternelj L, Bragard I, et al. Communication about uncertainty and hope: A randomized controlled trial assessing the efficacy of a communication skills training program for physicians caring for cancer patients. BMC Cancer 2017; 17:476.

14. Epstein AS, Prigerson HG, O'Reilly EM, et al. Discussions of Life Expectancy and Changes in Illness
Understanding in Patients With Advanced Cancer. J Clin Oncol 2016;34:2398-403.

15. Reblin M, Cloyes KG, Carpenter J, et al. Social support needs: discordance between home hospice nurses and former family caregivers. Palliat Support Care 2015;13:465-72.

16. Llombart-Cussac A, Pivot X, Biganzoli L, et al. A prognostic factor index for overall survival in patients receiving first-line chemotherapy for HER2-negative advanced breast cancer: an analysis of the ATHENA trial. Breast 2014;23:656-62.

17. Regierer AC, Wolters R, Ufen MP, et al. An internally and externally validated prognostic score for metastatic breast cancer: analysis of 2269 patients. Ann Oncol 2014;25:633-8.

18. Lee ES, Jung SY, Kim JY, et al. Identifying the potential long-term survivors among breast cancer patients with distant metastasis. Ann Oncol 2016;27:828-33.

19. Lee CK, Hudson M, Stockler M, et al. A nomogram to predict survival time in women starting first-line chemotherapy for advanced breast cancer. Breast Cancer Res Treat 2011;129:467-76.

20. Li S, Zhao J, Zhu L, et al. Development and validation of a nomogram predicting the overall survival of stage IV breast cancer patients. Cancer Med 2017;6:2586-94.

21. Genre L, Roché H, Varela L, et al. External validation of a published nomogram for prediction of brain metastasis in patients with extra-cerebral metastatic breast cancer and risk regression analysis. Eur J Cancer 2017;72:200-9.

22. Tominaga H, Setoguchi T, Shimada H, et al. Prognostic factors in patients with skeletal-related events at nonsmall-cell lung cancer diagnosis. Mol Clin Oncol 2017;7:897-902.

23. Filleron T, Bonnetain F, Mancini J, et al. Prospective construction and validation of a prognostic score to identify patients who benefit from third-line chemotherapy for metastatic breast cancer in terms of overall survival: the METAL3 Study. Contemp Clin Trials 2015;40:1-8.

24. Miao H, Hartman M, Bhoo-Pathy N, et al. Predicting survival of de novo metastatic breast cancer in Asian women: systematic review and validation study. PLoS One 2014;9:e93755.

25. Pagani O, Senkus E, Wood W, et al. International guidelines for management of metastatic breast cancer: can metastatic breast cancer be cured? J Natl Cancer Inst 2010;102:456-63.

26. Westphal T, Gampenrieder SP, Rinnerthaler G, et al. Cure in metastatic breast cancer. Memo 2018;11:172-9.

27. Gourd E. Pyrotinib shows activity in metastatic breast 
cancer. Lancet Oncol 2017;18:e643.

28. Ma F, Li Q, Chen S, et al. Phase I Study and Biomarker Analysis of Pyrotinib, a Novel Irreversible Pan-ErbB Receptor Tyrosine Kinase Inhibitor, in Patients With Human Epidermal Growth Factor Receptor 2-Positive Metastatic Breast Cancer. J Clin Oncol 2017;35:3105-12.

29. Goetz MP, Toi M, Campone M, et al. MONARCH 3 : Abemaciclib As Initial Therapy for Advanced Breast Cancer. J Clin Oncol 2017;35:3638-46.

30. Ellis MJ, Llombart-Cussac A, Feltl D, et al. Fulvestrant $500 \mathrm{mg}$ Versus Anastrozole $1 \mathrm{mg}$ for the First-Line Treatment of Advanced Breast Cancer: Overall Survival Analysis From the Phase II FIRST Study. J Clin Oncol 2015;33:3781-7.

Cite this article as: Zhao J, Yang Y, Pang D, Yu Y, Lin X, Chen K, Ye G, Tang J, Hu Q, Chai J, Bi Z, Ding L, Wu W, Zeng Y, Gui X, Liu D, Yao H, Wang Y. Development and validation of a nomogram in survival prediction among advanced breast cancer patients. Ann Transl Med 2020;8(21):1446. doi: 10.21037/atm-20-3473
31. Schmid P, Adams S, Rugo HS, et al. Atezolizumab and Nab-Paclitaxel in Advanced Triple-Negative Breast Cancer. N Engl J Med 2018;379:2108-21.

32. Kwapisz D. Oligometastatic breast cancer. Breast Cancer 2019;26:138-46.

33. Soran A, Ozmen V, Ozbas S, et al. Randomized Trial Comparing Resection of Primary Tumor with No Surgery in Stage IV Breast Cancer at Presentation: Protocol MF07-01. Ann Surg Oncol 2018;25:3141-9.

34. Subbiah IM, Lei X, Weinberg JS, et al. Validation and Development of a Modified Breast Graded Prognostic Assessment As a Tool for Survival in Patients With Breast Cancer and Brain Metastases. J Clin Oncol 2015;33:2239-45. 